MEROLLA Daniela and TURIN Mark (eds), 2017, Searching for Sharing. Heritage and Multimedia in Africa, Open Book Publishers, Cambridge UK, 150 p.

\title{
Cécile Leguy
}

\section{(2) OpenEdition}

Édition électronique

URL : https://journals.openedition.org/clo/5528

DOI : $10.4000 /$ clo.5528

ISSN : 2266-1816

Éditeur

INALCO

\section{Édition imprimée}

Date de publication : 5 décembre 2018

ISBN : 978-2-85831-338-9

ISSN : 0396-891X

\section{Référence électronique}

Cécile Leguy, « MERolta Daniela and turin Mark (eds), 2017, Searching for Sharing. Heritage and

Multimedia in Africa, Open Book Publishers, Cambridge UK, 150 p. », Cahiers de littérature orale [En ligne], 84 | 2018, mis en ligne le 05 décembre 2019, consulté le 23 mars 2022. URL : http:// journals.openedition.org/clo/5528; DOI : https://doi.org/10.4000/clo.5528

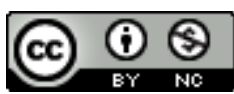

Cahiers de littérature orale est mis à disposition selon les termes de la Licence Creative Commons Attribution - Pas d'Utilisation Commerciale 4.0 International. 


\section{Merolla Daniela and Turin Mark (eds), 2017, Searching for Sharing. Heritage and Multimedia in Africa, Open Book Publishers, Cambridge UK, 150 p. (version en ligne : https://doi.org/10.11647/OBP.0111)}

Cécile Leguy

Université Sorbonne Nouvelle - Paris 3/CNRS-LACITO

Avec cet ouvrage au titre bien choisi, les chercheurs impliqués autour de Daniela Merolla et Mark Turin ne se contentent pas d'une réflexion sur le partage des connaissances et l'usage du multimédia concernant la littérature orale, ils mettent également leurs travaux à la portée de tout un chacun en publiant chez cet éditeur dont la politique générale est l'accès de tous au savoir. Ainsi, le livre est accessible dans son intégralité gratuitement en ligne, sur le site de l'éditeur. Pour les inconditionnels de la lecture sur support imprimé, il est aussi possible de le commander sous la forme d'un ouvrage dont la présentation, avec titres, intertitres et illustrations en couleurs, est de lecture très agréable.

L'ouvrage introduit par D. Merolla rassemble six chapitres portant sur différentes régions du continent africain dont Madagascar. Comme le rappelle M. Turin dans sa postface, le volume trouve son origine dans un projet de recherche dirigé par D. Merolla qui a rassemblé, à l'université de Rabat au Maroc en décembre 2013, les auteurs et d'autres participants autour d'une réflexion collective sur "Multimedia Research and Documentation of African Oral Genres : Connecting Diasporas and Local Audiences". L'objectif général du présent ouvrage est une réflexion sur les notions de partage, de partenariat, de restitution et de réappropriation dans le cadre des travaux sur les littératures orales, dimensions 
de la recherche qui ont pris une importance nouvelle avec le développement du multimédia. Plus précisément, l'objectif de ce livre est de penser le partage comme une méthode de travail, dans la mesure où il ne s'agit pas seulement pour le chercheur de présenter ses résultats aux populations concernées dans le cadre d'une restitution, mais bien de collaborer avec des acteurs sociaux, des artistes, des conteurs qui utilisent eux-mêmes les moyens modernes de communication pour valoriser leurs productions orales. Ainsi, la publication en open source défendue par les chercheurs trouve son pendant dans la diffusion sur le Net de productions relevant du patrimoine immatériel, soulevant des questionnements similaires en termes de droit à la propriété intellectuelle.

D. Merolla rappelle dans son introduction combien cette attention au dialogue entre les chercheurs et les acteurs du terrain est récente. Jean Rouch ${ }^{1}$ a dans ce domaine joué un rôle de précurseur, en développant dès les années 1960 une « anthropologie partagée », ayant le souci de présenter le résultat final de ses films documentaires dans les villages où il avait tourné et s'intéressant aux commentaires que cela provoquait. Mais ce sont surtout les débats autour de la question du rapatriement des pièces de musée (et en particulier des restes humains...) qui ont entraîné une réflexion plus générale sur la nécessaire collaboration et restitution des travaux vers les individus sur lesquels portent ceux-ci, comme le montre bien Jan Bender Shetler dans sa contribution sur « les implications du digital return sur la tradition orale » dans le contexte tanzanien. Aujourd'hui, grâce au développement du numérique, le « retour » (au sens de digital return) des données filmées, enregistrées sur le terrain, s'impose comme une évidence. Mais cela n'est pas sans poser de questions aux chercheurs, invités à s'interroger sur les conséquences que peuvent avoir ces données sur les populations concernées, notamment quand une production enregistrée est reçue en retour comme un « modèle ». Les débats menés au sujet de l'impact des travaux de l'équipe de Marcel Griaule sur la mythologie et les masques dogon sont bien connus (voir en particulier en français Doquet, 1999 ; Ciarcia, 2003), l'autorité scientifique du producteur (et le pouvoir des traces écrites laissées par les chercheurs : cf. Ciarcia \& Jolly, 2015) ayant contribué à modéliser ce qui fut ensuite présenté aux touristes comme « patrimoine culturel dogon ». Ainsi, les problèmes qui se posent ne sont pas seulement d'ordre juridique ou éthique ; la présentation que les chercheurs font des données qu'ils recueillent, passées au prisme de leurs options théoriques, ouvre aussi des débats sur la créativité et sur les risques de figement ou

1. Le Journal des africanistes a consacré son tome 87 au cinéaste-ethnologue (PECQUET, 2017). 
de standardisation. D. Merolla pose alors une question qui nous concerne tous ${ }^{2}$ : "Do [the researchers] participate in the 'heritagization' (a term we may derive from 'patrimonialisation' in French) of performances becoming museum pieces or tourist objects more than living social interactions?" (p. 9).

Par ailleurs, la mise à disposition de tous de productions parfois réservées à certaines catégories de personnes peut s'avérer délicate, notamment quand les performances enregistrées sont relatives à des rituels censés conserver une part de secret.

Cependant, comme le montrent bien les différentes contributions à cet ouvrage collectif, le partage peut aussi être compris comme une coopération, acteurs locaux et chercheurs œuvrant ensemble pour proposer des enregistrements documentés et contextualisés, les acteurs concernés pouvant également limiter les autorisations d'accès afin que certaines restrictions soient respectées (dans le cadre de savoirs propres à certaines catégories de personnes par exemple, ou quand les productions présentent des enjeux politiques ou religieux). La question se pose en effet de savoir non seulement ce qui peut être diffusé, mais aussi ce qui doit ou ne doit pas l'être. Le partage est bien une notion à comprendre dans sa dimension de réciprocité, non comme transfert de l'un à l'autre mais bien comme interaction. Le chercheur soucieux de ce qu'il diffuse doit se préoccuper de l'avis de tous les acteurs sociaux concernés pour s'assurer de la pertinence, mais aussi de l'innocuité, de ce qu'il partage, ce qui comme le fait bien remarquer D. Merolla (p. 13) peut lui demander du temps et de l'énergie. Il s'agit cependant de tenir compte des usages qui peuvent être faits des productions orales diffusées, en particulier dans une perspective éducative comme cela est envisagé dans différentes contributions à ce volume (voir entre autres le chapitre de Brahima Camara, Graeme Counsel et Jan Jansen sur l'utilisation de la chaîne YouTube comme outil pédagogique). Kofi Dorvlo, dénonçant l'action néfaste d'églises charismatiques locales sur la transmission culturelle, prône l'introduction, dans le programme scolaire, de productions orales et rituelles et la valorisation auprès du jeune public de manifestations culturelles comme celle du Hogbetsotso, un festival annuel institué en milieu ewe au Ghana depuis le début des années 1960. Russel Kaschula en contexte sud-africain et Valentin Vydrin en contexte mandingue (Mali-Guinée) présentent des archives digitales ambitieuses, ouvertes aux chercheurs comme aux usagers locaux. La

2. Cette problématique a été longuement discutée à l'occasion de l'exposition « Paroles d'Afrique » au musée d'Ethnographie de l'Université de Bordeaux (présentée au public du 23 octobre 2012 au 31 mai 2013) et lors du colloque éponyme auquel Daniela Merolla a participé (cf. DeRIVE \& Leguy, 2015 ; BornAND \& LeGUY, 2015 ; 2016). 


\section{CAHIERS DE LITTÉRATURE ORALE}

192 Le Récit généalogique $-n^{\circ} 84$

réflexion menée par Brigitte Rasoloniaina et Andriamanivohasina Rakotomalala sur le « retour » des travaux de trois générations de chercheurs aux objectifs divers (intellectuels locaux, missionnaires, chercheurs du temps de la colonisation...) interroge l'impact de leur usage, notamment des recueils de contes malgaches ou européens utilisés au cours du temps dans les écoles, et leur influence sur les productions postérieures.

Comme le rappelle Mark Turin dans sa postface, si "Searching for Sharing is situated in a rich intellectual tradition that has long explored issues of orality, textuality, performance and cultural heritage" (p. 144), les opportunités ouvertes par le digital turn laissent envisager de nouvelles relations de coopération et de dialogue entre les différents acteurs d'une recherche partagée et responsable.

\section{Références citées :}

Bornand Sandra \& Leguy Cécile, 2015, « Des "paroles d'Afrique" dans un musée : de la valorisation à la transmission », in Sur les pas de Geneviève Calame-Griaule, Journal des Africanistes, no 85-1/2, p. 44-78.

Bornand Sandra \& Leguy Cécile, 2016, "Exhibiting Intangible Heritage in a Museum: The Voices of Africa Experience", in Nordic Journal of African Studies, n 25, vol. 3-4, pp. 364-384.

Ciarcia Gaetano, 2003, De la Mémoire ethnographique. L'exotisme du pays dogon, Éditions de l'EHESS, Paris, 200 p.

Ciarcia Gaetano \& Jolly Éric (dir.), 2015, Métamorphoses de l'oralité entre écrit et image, Karthala, Paris, 288 p.

Derive Jean \& Leguy Cécile (dir.), 2015, Paroles d'Afrique in Cargo. Revue internationale d'anthropologie culturelle \& sociale, $\mathrm{n}^{\circ} 4$.

Dopuet Anne, 1999, Les Masques dogon. Ethnologie savante et ethnologie autochtone, Karthala, Paris, 320 p.

Pecquet Luc (dir.), 2017, Jean Rouch, ethnologue et cinéaste, Journal des Africanistes, $\mathrm{n}^{\circ} 87-1 / 2$. 